\title{
Mitigating Implicit Bias in Patient-Clinician Communication in Clinical Encounters: Protocol from the COmmuNity-engaged SimULation Training (CONSULT) Trial
}

Jennifer Tjia ( $\square$ Jennifer.tjia@umassmed.edu )

University of Massachusetts Medical School https://orcid.org/0000-0003-4541-0460

Michele Pugnaire

University of Massachusetts Medical School

Joanne Calista

Center for Health Impact

Nancy Esparza

Center for Health Impact

Olga Valdman

University of Massachusetts Medical School

Maria Garcia

University of Massachusetts Medical School

Majid Yazdani

University of Massachusetts Medical School

Janet Hale

University of Massachusetts Medical School

Jill Terrien

University of Massachusetts Medical School

\section{Ethan Eisdorfer}

University of Massachusetts Medical School

Valerie Zolezzi-Wyndham

Promoting Good

Germán Chiriboga

University of Massachusetts Medical School

Geraldine Puerto

University of Massachusetts Medical School

\section{Elizabeth Dykhouse}

University of Massachusetts Medical School

\section{Stacy Potts}

University of Massachusetts Medical School 


\section{Andriana Foiles Sifuentes}

University of Massachusetts Medical School

\section{Sylvia Stanhope}

University of Massachusetts Medical School

Jeroan Allison

University of Massachusetts Medical School

Vennesa Duodu

University of Massachusetts Medical School

Janice Sabin

University of Washington

\section{Study protocol}

Keywords: implicit bias, medical education, bias awareness, community engagement, simulation training

Posted Date: October 28th, 2020

DOl: https://doi.org/10.21203/rs.3.rs-39615/v2

License: (c) (1) This work is licensed under a Creative Commons Attribution 4.0 International License.

Read Full License 


\section{Abstract}

Background: Healthcare professionals have negative implicit biases toward minority and poor patients. Few communication skills interventions target implicit bias as a factor contributing to disparities in health outcomes. We report the protocol from the COmmuNity-engaged SimULation Training for Blood Pressure Control (CONSULT-BP), a trial evaluating a novel intervention targeting graduate medical and nursing trainees designed to mitigate the effects of implicit bias in clinical encounters. The CONSULTBP intervention combines knowledge acquisition, bias awareness, and practice of bias mitigating skills in simulation-based communication encounters with racially/ethnically diverse standardized patients. The trial evaluates the effect of this 3-part program on patient BP outcomes, self-reported patient medication adherence, patient-reported quality of provider communication, and trainee bias awareness.

Methods: We are conducting a cluster randomized trial of the intervention among cohorts of internal medicine (IM), family medicine (FM), and doctorate nurse practitioner (DNP) trainees at a single academic medical center. We are enrolling entire specialty cohorts of IM, FM, and NP trainees over a 3year period, with each academic year constituting an intervention cycle. There are 3 cycles of implementation corresponding to 3 sequential academic years. Within each academic year, we randomize training times to 1 of 5 start dates using a stepped wedge design. The stepped wedge design compares outcomes within training clusters before and after the intervention, as well as across exposed and unexposed clusters. Primary outcome of blood pressure control is measured at the patient-level for patients clustered within trainees. Eligible patients for outcomes analysis are: English-speaking; nonWhite racial/ethnic minority; Medicaid recipient (regardless of race/ethnicity); hypertension; not have pregnancy, dementia, schizophrenia, bipolar illness, or other serious comorbidities that would interfere with hypertension self-control; not enrolled in hospice. Secondary outcomes include trainee bias awareness. A unique feature of this trial is the engagement of academic and community stakeholders to design, pilot test and implement a training program addressing healthcare.

Discussion: Equipping clinicians with skills to mitigate implicit bias in clinical encounters is crucial to addressing persistent disparities in healthcare outcomes. Our novel, integrated approach may improve patient outcomes.

Trial registration: NCT03375918

Protocol version : 2.0 (October 16, 2020)

\section{Introduction}

\section{Rationale for the Trial}

Evidence suggests that healthcare professionals have negative explicit and implicit biases toward minority and poor patients, ${ }^{1,2}$ which can adversely affect clinical decision-making ${ }^{3-6}$ and interpersonal 
communication. ${ }^{7}$ Mitigating clinician bias through targeted training in bias awareness and interpersonal communication skills is a promising strategy for decreasing healthcare disparities for racially, ethnically, and socio-economically disadvantaged persons. ${ }^{8}$ Few rigorously designed trials of communication skills target implicit bias as a factor contributing to disparities in health outcomes. ${ }^{9,10}$

To address this gap, we designed a theoretically-grounded, multi-component, training intervention trial called "COmmuNity-engaged SimULation Training for Blood Pressure Control" (CONSULT-BP). The primary aim of the trial is to improve patient outcomes using a training program that combines knowledge acquisition, bias awareness development, and simulation-based communication practice with racially and ethnically diverse patients in order to better prepare clinicians for patient interactions in which implicit bias may affect healthcare outcomes. The clinical focus of the intervention is hypertension management because it represents a challenging public health priority, ${ }_{1}^{11,12}$ with healthcare disparities and clinical outcomes mediated, in part, by the quality of clinician-patient communication, bias $^{13}$ and unsatisfactory patient experiences of care. ${ }^{14}$

The current educational arsenal to address racism and bias in healthcare has critical gaps, notably failing to incorporate personal bias awareness and evidence-based bias mitigating strategies into a program of practice and feedback from patients. ${ }^{10}$ We address these gaps in our trial by implementing a multi-component "knowledge, awareness, and practice" training model that involves community member and patient representation during the design and operationalization of the educational intervention. This protocol report provides a resource for others seeking to design a trial that evaluates and implements a training intervention to mitigate implicit bias in clinician-patient encounters.

\section{Objectives}

The aims of the clinical trial are to evaluate the effect of this 3-part, training program on: 1. patient blood pressure outcomes, reported in the electronic medical record (EMR); 2. self-reported patient medication and diet adherence, as measured by the Hypertension Medication Nonadherence Scale ${ }^{15}$ and the Blood Pressure Self-Care Scale; ${ }^{16} 3$. patient-reported quality of provider communication, as measured by the Health Care Climate Questionnaire; ${ }^{17} 4$. the trust sub-scale of the Primary Care Assessment Survey; ${ }^{18}$ and 5. trainee bias awareness, as measured by the Bias Awareness Scale. ${ }^{19}$

\section{INTERVENTION}

\section{Description of the CONSULT-BP Educational Intervention}

\section{$\underline{\text { Core Educational Elements }}$}

The three (3) core elements of the educational intervention include: 1. trainee knowledge acquisition about healthcare disparities, implicit bias and racism; 2 . trainee awareness of personal bias; and 3 . provision of skills to mitigate bias, along with an opportunity to practice those skills with authentic standardized patient actors. A Standardized Patient (SP) is a person carefully recruited and trained to 
take on the characteristics of a real patient thereby affording learners an opportunity to practice and be evaluated on learned skills in a simulated clinical environment.

To build knowledge, we used National Institute of Minority Health and Health Disparities-funded elearning modules about health disparities, implicit bias, and patient-centered communication skills. ${ }^{20} \mathrm{We}$ also deliver evidence-based practice knowledge about hypertension management, because the skills practice cases with SPs focus on hypertension management. To develop personal bias awareness, we use the Implicit Association Tests (IAT) ${ }^{21}$ with results feedback to the trainee. To develop skills, we present evidence-based strategies to mitigate bias previously tested in a randomized trial by Devine et al. ${ }^{22}$ Finally, trainees are provided an opportunity to practice those bias-mitigating skills using high-fidelity simulated clinical encounters with SPs. ${ }^{23}$ (Figure 1)

\section{Theoretical Framework}

The educational intervention and its delivery were designed to reflect key features of an adaptation of Bennett's intercultural competency framework. ${ }^{24,25}$ (Figure 2) In this theoretical model, to overcome denial about ones' own implicit biases, learners need to first acquire knowledge and understanding about implicit bias. Then, to help learners develop acceptance of the effect of implicit bias on healthcare disparities, learners need to move toward integration and recognition of implicit bias within themselves and their clinical encounters. The ultimate goal is to motivate learners to acquire and apply effective communication skills in situations where implicit bias is likely to arise.

Applying the simulation-based model of repeated practice and feedback for skills acquisition and progression to skill mastery, ${ }^{26}$ the CONSULT-BP training featured "mock" clinical encounters designed to "activate" trainee biases in the clinical care setting. To replicate the "authentic" experience of implicit bias in clinical care, the CONSULT-BP intervention developed face-to-face simulated clinical encounters with "acting" SPs recruited from local racial and ethnic communities as a foundational component of the program. These face-to-face interactions provided a "contact-based educational intervention." ${ }^{25}$ Contact with groups for which one may hold biased attitudes may help reduce such bias. ${ }^{22,27}$

\section{Methods And Analysis}

\section{Trial Design}

This is a cluster randomized trial of the CONSULT-BP training using a stepped wedge design to evaluate the effectiveness of one-time CONSULT-BP training on patient outcomes. The stepped wedge design accommodates pre-existing training schedules to mitigate the effect of temporal trends in clinical skill proficiency. It also allows for all trainees to be assigned to the intervention, which was a precondition of involvement by the participating training programs, in order to meet training requirements. This design is statistically advantageous because all trainees have control and intervention periods, everyone serves as their own control, allowing for within and across participant comparisons based on 
data collection both before and after the intervention. ${ }^{28}$ Our model randomizes training times to one of five start dates within each academic year to accommodate pre-existing training schedules and to mitigate the effect of temporal trends in clinical skill proficiency.

\section{Setting}

This trial is being conducted at a single academic safety net hospital system that includes clinical delivery sites at an academic medical center and a community hospital. Approximately $40 \%$ of medical center's primary care patients are low income and $\sim 27 \%$ non-white. The surrounding community includes $34 \%$ of residents that do not speak English at home, $21 \%$ are Hispanic/Latino, and there are large newcomer immigrant and refugee populations. The medical center is a major regional training site for medical students, graduate nursing students, and postgraduate medical residents. The participating training programs include the internal medicine (IM), family medicine (FM) residency training programs, and the doctor nurse practitioner (DNP) graduate school program. Participant recruitment started in September 2018 and we will enroll trainees and patients until August 2021. Data collection will continue until November 2021.

\section{PARTICIPANTS}

\section{Eligibility Criteria}

\section{Trainee Enrollment}

Training programs assign their own trainees to participate in the intervention. Eligibility criteria for inclusion of trainee measures in the trial's outcomes analysis are: 1. practice at a clinical site supported by the medical center's EMR to allow data collection for BP outcome measurement; 2. a 15-week clinical look-back period; and 3. no prior completion of the CONSULT-BP intervention. Trainees are provided a fact sheet and asked to opt-out of the study if they do not want their data used for outcomes analysis.

\section{Patient Enrollment}

Primary outcomes of blood pressure control are measured at the patient level for patients clustered within trainees as reported in the electronic medical record. Patients eligible for inclusion in the outcome analysis included being: 1. English-speaking; 2 . Non-White racial/ethnic minority; 3 . Medicaid recipient (regardless of race/ethnicity); 4. have hypertension. Exclusion criteria: We exclude patients with pregnancy, dementia, schizophrenia, bipolar illness, or other serious medical co-morbidity that would interfere with hypertension self-control, including enrollment in hospice. Secondary patient-reported outcomes of self-reported adherence and quality of communication with providers is measured from a subset of patients meeting the same eligibility criteria who are randomly recruited in periods before and after the training intervention.

\section{Intervention Assignment and Recruitment}


The educational intervention was integrated into the residency programs in IM and $\mathrm{FM}$, and into the curriculum for DNP students. We are enrolling entire specialty cohorts of IM, FM, and NP trainees over a 3-year period, with each academic year constituting an intervention cycle. There are 3 cycles of implementation corresponding to 3 sequential academic years. Within each academic year, we randomize training times to 1 of 5 start dates using a stepped wedge design. Our biostatistician uses a random sequence generator to assign 5 groups of learners, from within each program, to 1 of 5 staggered intervention training dates within an academic year. Training program schedulers assign the trainees according to these assigned dates. The program is delivered over two in-person sessions, scheduled five weeks apart, to leverage a spaced learning design. ${ }^{29}$ The sessions were designed to minimize the time burden of the intervention on trainees outside of the classroom. As such, the in-person training sessions combined multiple educational components that trainees completed individually, but as part of an onsite group session. In each educational session, components included: "individual" online didactic modules, online IATs, face-to-face clinical practice simulation with SPs, and "group-based" facilitated debriefing sessions. Skills to mitigate the effects of implicit bias detailed by Devine et al ${ }^{30}$ were addressed as part of the group-based IAT debriefings that preceded case simulations with SPs. Each of the two, in-person sessions lasted about four hours. If trainee participants were unable to attend one of the sessions due to scheduling conflicts, we provided alternative dates for attendance.

\section{Outcome Measures and Data Collection}

\section{Trainee Measures}

Trainee Demographics. We collect trainee age, gender, race/ethnicity, training year, whether they are US or foreign-born, and fluency in another language.

Trainee IAT, Explicit Bias Measures, Bias Awareness, and Reaction to the IAT. Trainees are asked to complete online Race/Ethnicity IATs [Black/White, Latino/White] ${ }^{21}$ and Race/Ethnicity-Medical Compliance IATs [Black/White, Latino/White]. ${ }^{31}$ Trainees are asked about their own explicit beliefs and perceptions of what "other health professionals" believe about race/ethnicity and race/ethnicity-related medical compliance in order to assess trainees' perception of their own bias as being "better than average". ${ }^{32}$ To assess trainees' reaction to the IAT, three questions are included from Howell \& Ratliff ${ }^{32}$ using a 4-point scale (Strongly disagree, Disagree, Agree, Strongly Agree) to measure the trainees' degree of defensiveness to the IAT. Trainees also complete a 7-item Bias Awareness Scale, with items assessed on a 6-point scale (strongly agree to strongly disagree) and higher scores indicating greater bias awareness. ${ }^{19}$ These items were collected online during each of the in-person training sessions, including 2 IATs at each session.

Assessment of Trainees by SPS. Community SPs complete standardized checklists of trainee performance measuring communication skills, perceptions of respect, emotional response, concern, empathy, listening skills, involvement in shared decision-making, engagement and partnership, BP measurement technique, and global performance. 
The primary trial outcome is the change in BP as reported in the EMR. Clinical measurements are collected in the 15-week control period prior to CONSULT-BP training period and during a 20-week post intervention period. The main outcome measures in the primary analysis will be systolic and diastolic BP $(\mathrm{mm} \mathrm{Hg})$ from the EMR. Analyses will stratify BP control as defined by the Joint National Commission 833: controlled HTN defined as SBP of $<140 / 90$ for most patients, or SBP $<150 / 90$ for patients $\geq 60$ years without a diagnosis of diabetes or chronic kidney disease, and uncontrolled hypertension as defined as greater than these values.

Secondary outcomes include patient self-reported adherence to visits, diet modification and antihypertensive medication use as measured by the BP Self-Care Scale, ${ }^{16}$ Hypertension Medication Nonadherence Scale, ${ }^{15}$ and quality of communication and trust measured in the Health Care Climate Questionnaire ${ }^{17}$ and the trust sub-scale of the Primary Care Assessment Survey. ${ }^{18}$ These surveys are administered in clinic offices of participating trainees in the 15-weeks before and 20-week after the educational intervention. Following the stepped wedge design, all comparisons are before and after the intervention within patients nested within trainee and across trainees. Trained research assistants collect primary data from of 5 patients per trainee in both the pre- and post-intervention periods. Patients are asked to rate the clinical interaction with the trainee on that day. Pictures of residents are included at the beginning of the questionnaire to confirm that the responses are being provided for the clinical trainee pictured.

\section{Data Management}

We conducted data abstractions from the electronic medical record for primary outcomes assessment using a system that extracts data from the clinical database, reformats it and stores it on a separate analytics server to make querying the database more efficient and extract data without impacting clinical flow. Primary data collection from trainee self-report and patient questionnaires were entered into an electronic data capture system, RedCAP, using case report forms designed for this purpose, with the exception of the IATs which were directly collected by Project Implicit via an internetbased interface. Standardized patient assessments of trainee performance in the simulation lab are entered into the Learning Space data capture system used for all training and evaluation exercises at the clinical simulation lab.

\section{Statistical analysis}

For the primary outcome, we will compare the SBP and DBP of patients to be seen by the participant trainees in the control period vs. the period after the training. For visits with more than one BP measurement, we will average the measurements within the visit. We will first compare the mean difference in SBP and DBP between the two study periods using mixed effects models (Model 1). We will then examine the linear trend of intervention effects using another set of mixed effect models (Model 2). Both models will adjust for patient and clinician characteristics, and include multi-level random effects to 
account for the correlation among patients in the same clusters of randomization, patients nested within clinicians and repeated measures from the same patients (same patients can be seen by the same clinician at control and after-training periods). Finally, we will conduct subgroup analysis by repeating the same analyses stratified by uncontrolled and controlled hypertension at baseline based on the definition in JNC $8 .{ }^{33}$

Secondary outcomes examine the role of trainee bias awareness and IAT on patient outcomes. We hypothesize that lower implicit bias and higher bias awareness are associated with lower postintervention patient BP and better patient reported communication scores. The main outcomes for this analysis are patient reported outcomes (PROs) after the CONSULT-BP training. The main independent variables are trainees' IATs (1. race and 2. race-compliance used in separate models) and bias awareness. The following mixed effects linear model (Model 3) will be used to examine the association between post- training PROs scores and clinicians' IATs, adjusting for patient and clinician characteristics, and the correlation among patients nested in clinician (random effect). We will also control for the pretraining PRO score in the model. Since the pre-training score for each post-training patient is not available we will use the mean PRO score collected from the patients seen by clinician before training as a surrogate for the pre-training score. We will use separate models for race IAT and race-compliance IATs, stratified by Black-White race and Latino/Hispanic-White race comparisons in separate models.

Descriptive analyses will characterize missing data frequency and associations with key trainee and patient characteristics. Variables associated with missingness will be included in analyses as covariates to account for effects from the missing pattern (missing at random).

\section{Sample size calculation}

For the primary outcome of BP change, we estimate that each resident will have at least 5 eligible patients per 5-week training block (assuming trainees see 25 patients per outpatient rotation block, of whom $\sim 40 \%$ have HTN ( $n \sim 10)$, and of whom $\sim 45 \%$ are low income). Thus, each trainee will see $\sim 10$ eligible patients in the 3-block pre-intervention period, and $\sim 20$ eligible patients in the 4 block postintervention period. We estimate there will be approximately 205 enrolled trainees over a 3 year accrual period, yielding $\sim 205 \times 10$ pre-intervention observations $(\mathrm{n}=2050)$ and $205 \times 20$ post-intervention observations $(n=4100)$ for all patients with HTN, and fewer (control 1025 and intervention 2050) for

participants with uncontrolled HTN (50-70\% in minority and poor populations). ${ }^{34}$ We will have $>90 \%$ power to detect a $3 \mathrm{mmHg}$ difference in patients at control and post-training periods.

\section{Patient and Public Involvement - Development of the Intervention Content and Design}

A unique feature of this trial was the engagement of academic and community stakeholders to design, pilot test and implement a training program addressing healthcare. To adapt the core educational elements and strategies into a feasible and acceptable educational intervention, we used a participation action research approach in which investigators collaboratively partnered with stakeholder participants. The goal was to work together to address system-specific issues affecting program 
operationalization. ${ }^{35}$ Community stakeholder partners for educational design and delivery were $\mathrm{racial} /$ ethnic and socioeconomically diverse community leaders representing our local patient population. A local community health organization, with whom the study team had a long-standing research relationship, served as the community member recruitment liaison. Key academic stakeholder partners for educational design were School of Medicine and Graduate School of Nursing faculty from our target healthcare system. A separate community-based transformational change organization, with extensive experience in staff bias training and who worked closely with the medical center administrative leadership, was engaged to help design in-person facilitation around implicit bias. These partners worked together to design case simulation scenarios, a trainee performance evaluation checklist, and SP training protocols that were integrated into a cohesive, replicable training program. Finally, community members were recruited to be SPs, which created an opportunity for empowerment and equity, as community SP's provided direct skills feedback to healthcare trainees and contributed their "voice" as equal partners in the team effort to develop and refine the simulation scenarios. We sought to understand whether this creative and novel approach to communication skills training of healthcare professionals catalyzed the motivation of our learners to take their patient communication skills to a higher level of mastery through direct, objective, and specific feedback from individuals of color trained as SPs. ${ }^{25,36,37}$

\section{Monitoring}

The PI, in cooperation with the coinvestigators and the UMass Medical School Institutional Review Board, are monitoring the safety of the proposed project. We have created a data safety and monitoring plan and established formal monitoring procedures to closely monitor participate safety, data quality and study progress. A data monitoring committee is not needed since the study is minimal risk.

Monitoring for protocol adherence will be performed monthly to ensure early identify of poor performance. Specific parameters being monitored include psychological harm from the intervention, contacting patients for surveys who are ineligible for the study; all adverse events are reported to the IRB. Our study protocol includes informed consent of the trainees for inclusion of their data in the outcome analysis, and informed consent for the patient questionnaires, and a Health Insurance Portability and Accountability Act (HIPPA) waiver for use of medical record data in the aggregated outcomes analysis.

\section{ETHICS AND DISSEMINATION}

\section{Safety Concerns}

We obtained Institutional Review Board (IRB) approval at the UMass Medical School. To minimize research-associated risk and to protect the confidentiality of participant data, all investigators and staff involved in this project have completed extensive courses and passed certifying examination on the protection of human subjects in research through Collaborative Institutional Training Initiative training and HIPPA certification. The IRB conducts interim audits per their policies and procedures to assure compliance with standards of trial implementation. 


\section{Dissemination plan}

Patient and organizational stakeholder will significantly contribute to the translation of the research findings into lay language. Additionally, our group has long-standing relationships with large, community-based organizations and will work closely with these stakeholders to develop and activate the infrastructure to dissemination our results through community-based outreach and academic venues. As per funder requirements, we will provide limited access to the final trial dataset via contractual agreements assuring data protections and security procedures. Furthermore, the protocol has been, and the results of this study will be, submitted to ClinicalTrials.gov and peer-review publications.

\section{Discussion}

In approaching the task of implicit bias training in healthcare, a gap exists between what is theoretically known to be effective. ${ }^{24,25}$ Appreciation of evidence that implicit bias exists in healthcare professionals has arguably been one of the most challenging aspects of addressing the persistent gaps in healthcare disparities. As modeled by Bennett and others, ${ }^{24,25}$ recognition and self-awareness of bias are the critical first steps in implicit bias training. Building on these priorities, the CONSULT-BP program specifically targets these first stages in the learning process of bias mitigation for healthcare professions.

Our effort to implement theoretically sound and novel approaches to clinician skill building for bias mitigation and patient-clinician communication across intercultural differences holds important implications for public health. While CONSULT-BP focuses on hypertension, the CONSULT-BP model is readily adaptable to other diseases, such as COVID-19, in which decisions for testing are emerging as being subject to bias. ${ }^{38}$ This adaptability of the CONSULT-BP model is an important as disparities in healthcare access and outcomes for racial, ethnic and poor populations is a persistent problem. Further, the Centers for Disease Control and Prevention recommends bias awareness and mitigation skills to address the public health crisis of COVID-19. ${ }^{39}$ To date, the domain of educational interventions for bias training remains ripe for development and the preliminary findings from the CONSULT-BP program contribute useful insights into the design of bias training models for healthcare professionals. Our study provides a roadmap for others who share these goals and offers lessons learned that others can use to advance their own efforts.

\section{Declarations}

ACKNOWLEDGEMENTS We would like to thank the community advisors, standardized patients, and trainees for their generous contributions to this project.

DISCLOSURES OF CONFLICT The authors have no competing interests to disclose.

CONTRIBUTORS JT: conception and design of study, acquisition of study data, drafting and critically revising the manuscript; NE, JH, LR, JT, JC, GC, GP, VD, SS, AFS: Acquisition of study data, drafting and 
critically revising the manuscript; MP, OV, MG, MY, ED, EE, SP: conception and design of study, critically revising the manuscript; JS: conception and design of study, drafting and critically revising the manuscript.

FUNDING SOURCE Research reported in this manuscript was supported by the National Institute of Minority Health and Health Disparities of the National Institutes of Health under award number R01MD011532. The funder had no role in the study design, collection, management, analysis and interpretation of data; writing of the report; and the decision to submit the protocol for publication.

DISCLAIMER The content is solely the responsibility of the authors and does not necessarily represent the official views of the National Institutes of Health.

NAME OF IRB GRANTING INSTITITION University of Massachusetts Medical School

PRIOR PRESENTATIONS An earlier version of this paper was presented at the $12^{\text {th }}$ Annual Conference on the Science of Dissemination and Implementation, Arlington, VA. December 2019

\section{References}

1. Maina I, Belton T, Ginzberg S, Singh A, Jjohnson T. A decade of studying implicit racial/ethnic bias in healthcare providers using the implicit associaiton test. Soc Sci Med. 2018;199:219-229.

2. FitzGerald C, Hurst S. Implicit bias in healthcare professionals: a systematic review. BMC Med Ethics. 2017;18(1):19.

3. Green AR, Carney DR, Pallin DJ, et al. Implicit bias among physicians and its prediction of thrombolysis decisions for black and white patients. J Gen Intern Med. 2007;22(9):1231-1238.

4. Penner LA, Dovidio JF, West TV, Gaertner SL, Albrech TL. Aversive racism and medical interactions with black patients: A field study. Journal of Experimental Psychology. 2010;46(2):436-440.

5. Sabin JA, Greenwald AG. The influence of implicit bias on treatment recommendations for 4 common pediatric conditions: pain, urinary tract infection, attention deficit hyperactivity disorder, and asthma. Am J Public Health. 2012;102(5):988-995.

6. Bogart LM, Catz SL, Kelley JA, Benotsch EG. Factors influencing physicians' judgments of adherence and treatment decisions for patients with HIV disease. Medical Decision Making. 2001;21(1):28-36.

7. Cooper LA, Roter DL, Carson KA, et al. The associations of clinicians' implicit attitudes about race with medical visit communication and patient ratings of interpersonal care. Am J Public Health. 2012;102(5):979-987.

8. Smedley B, Stith A, Nelson A. Unequal treatment: Confronting racial and ethnic disparities in health care. Washington, D.C.: National Academy Press; 2003.

9. Cooper LA, Roter DL, Bone LR, et al. A randomized controlled trial of interventions to enhance patientphysician partnership, patient adherence and high blood pressure control among ethnic minorities and poor persons: study protocol NCT00123045. Implement Sci. 2009;4:7. 
10. Beach MC, Gary TL, Price EG, et al. Improving health care quality for racial/ethnic minorities: a systematic review of the best evidence regarding provider and organization interventions. $B M C$ Public Health. 2006;6:104.

11. Fisher NDL, Curfman G. Hypertension-A Public Health Challenge of Global Proportions. JAMA. 2018;320(17):1757-1759.

12. US Department of Health and Human Services. The Surgeon General's Call to Action to Control Hypertension. Washington, DC: Office of the Surgeon General, U.S. Department of Health and Human Services;2020.

13. Schoenthaler A, Chaplin WF, Allegrante JP, et al. Provider communication effects medication adherence in hypertensive African Americans. Patient Educ Couns. 2009;75(2):185-191.

14. Hanlin RB, Asif IM, Wozniak G, et al. Measure Accurately, Act Rapidly, and Partner With Patients (MAP) improves hypertension control in medically underserved patients: Care Coordination Institute and American Medical Association Hypertension Control Project Pilot Study results. J Clin Hypertens (Greenwich). 2018;20(1):79-87.

15. Voils Cl, Maciejewski ML, Hoyle RH, et al. Initial validation of a self-report measure of the extent of and reasons for medication nonadherence. Medical care. 2012;50(12):1013-1019.

16. Peters RM, Templin TN. Measuring blood pressure knowledge and self-care behaviors of African Americans. Res Nurs Health. 2008;31(6):543-552.

17. Kasser VG, Ryan RM. The Relation of Psychological Needs for Autonomy and Relatedness to Vitality, Well-Being, and Mortality in a Nursing Home1. Journal of Applied Social Psychology. 1999;29(5):935954.

18. Safran DG, Kosinski M, Tarlov AR, et al. The Primary Care Assessment Survey: tests of data quality and measurement performance. Med Care. 1998;36(5):728-739.

19. Girod S, Fassiotto M, Grewal D, et al. Reducing Implicit Gender Leadership Bias in Academic Medicine With an Educational Intervention. Acad Med. 2016.

20. Van Schaik E, Howson A, Sabin J. Healthcare Disparities. 2014(9675). www.mededportal.org/publication/9675.

21. Greenwald AG, McGhee DE, Schwartz JL. Measuring individual differences in implicit cognition: The Implicit Association Test. Journal of Personality and Social Psychology. 1998;74(6):1464-1480.

22. Devine $P$, Forscher $P$, Austin A, Cox W. Long-term reductionin implicit race bias: A prejudice habitbreaking intervention. J Exp Soc Psychol. 2012;48(6):1267-1278.

23. Ziv A, Wolpe PR, Small SD, Glick S. Simulation-based medical education: an ethical imperative. Simul Healthc. 2006;1(4):252-256.

24. Bennett MJ. A developmental approach to training for intercultural sensitivity. International Journal of Intercultural Relations. 1986;10(2):179-196.

25. Teal CR, Gill AC, Green AR, Crandall S. Helping medical learners recognise and manage unconscious bias toward certain patient groups. Med Educ. 2012;46(1):80-88. 
26. Shute VJ, Gawlick LA. Practice Effects on Skill Acquisition, Learning Outcome, Retention, and Sensitivity to Relearning. Human Factors. 1995;37(4):781-803.

27. Dasgupta N, Greenwald AG. On the malleability of automatic attitudes: Combating automatic prejudice with images of admired and disliked individuals. J of Personality and Social Psychology. 2001;81(5):1-15.

28. Hemming K, Haines T, Chilton P, Girling A, Lilford R. The stepped wedge cluster randomized trial: rationale, design, analysis, and reporting. BMJ. 2015;350(h391-h391).

29. Kerfoot BP, Fu Y, Baker H, Connelly D, Ritchey ML, Genega EM. Online spaced education generates transfer and improves long-term retention of diagnostic skills: a randomized controlled trial. J Am Coll Surg. 2010;211(3):331-337 e331.

30. Devine PG, Forscher PS, Austin AJ, Cox WTL. Long-term reduction in implicit race bias: A prejudice habit-breaking intervention. Journal of experimental social psychology. 2012;48(6):1267-1278.

31. Sabin JA, Rivara FP, Greenwald AG. Physician implicit attitudes and stereotypes about race and quality of medical care. Med Care. 2008;46(7):678-685.

32. Howell JL, Ratliff KA. Not your average bigot: The better-than-average effect and defensive responding to Implicit Association Test feedback. Br J Soc Psychol. 2017;56(1):125-145.

33. James PA, Oparil S, Carter BL, et al. 2014 evidence-based guideline for the management of high blood pressure in adults: report from the panel members appointed to the Eighth Joint National Committee (JNC 8). JAMA. 2014;311(5):507-520.

34. Gillespie C, Hurvitz K. Prevalence of Hypertension and Controlled Hypertension - United States, 2007-2010. MMWR - Suppl. 2013;62:144-148e.

35. Leykum LK, Pugh JA, Lanham HJ, Harmon J, McDaniel RR, Jr. Implementation research design: integrating participatory action research into randomized controlled trials. Implement Sci. 2009;4:69.

36. Johnson RL, Roter DL, Powe NR, Cooper LA. Patient race/ethnicity and quality of patient-physician communication during medical visits. American Journal of Public Health. 2004;94(12):2084-2090.

37. Morell VW, Sharp PC, Crandall SJ. Creating student awareness to improve cultural competence: creating the critical incident. Med Teach. 2002;24(5):532-534.

38. Rubix Life Sciences. COVID-19 and Minority Health Access.; 2020. https://rubixls.com/wpcontent/uploads/2020/04/COVID-19-Minority-Health-Access-7-1.pdf. Accessed May 21, 2020.

39. Eligon J, Burch A. Questions of Bias in COVID-19 Treatment Add to the Mourning for Black Families. The New York Times. May 10, 2020, 2020.

\section{Table}

\section{Table 1. COmmuNity-engaged SimULation Training for Blood Pressure Control (CONSULT-BP): Core elements and Tailored Elements}




\begin{tabular}{|c|c|c|}
\hline \multicolumn{2}{|r|}{ CORE ELEMENTS } & TAILORED ELEMENTS \\
\hline $\begin{array}{c}\text { Key } \\
\text { Domains }\end{array}$ & Key Content & CONSULT-BP \\
\hline \multicolumn{2}{|c|}{$\begin{array}{l}\text { Knowledge } \\
\text { : Healthcare Disparities } \\
\text { - Implicit Bias } \\
\text { C } \$ \text { mmunication Skills to Mitigate Implicit Bias }\end{array}$} & $\begin{array}{l}\text { Online, self-administered, } \\
\text { learning modules }\end{array}$ \\
\hline $\begin{array}{l}\text { Self- } \cdot \text { In } \\
\text { Awareness }\end{array}$ & plicit Bias & $\begin{array}{l}4 \text { IATs } \\
\text { ace-bias (2) } \\
\text { ace-compliance bias (2) }\end{array}$ \\
\hline $\begin{array}{ll}\text { Skills } & \\
\text { Practice } & \mathrm{H} \\
\mathrm{fr}\end{array}$ & $\begin{array}{l}\text { pertension management case with patient } \\
\text { pm vulnerable population }\end{array}$ & $\begin{array}{l}4 \text { standardized patient } \\
\text { encounters }\end{array}$ \\
\hline
\end{tabular}

IAT = Implicit Association Test

\section{Figures}




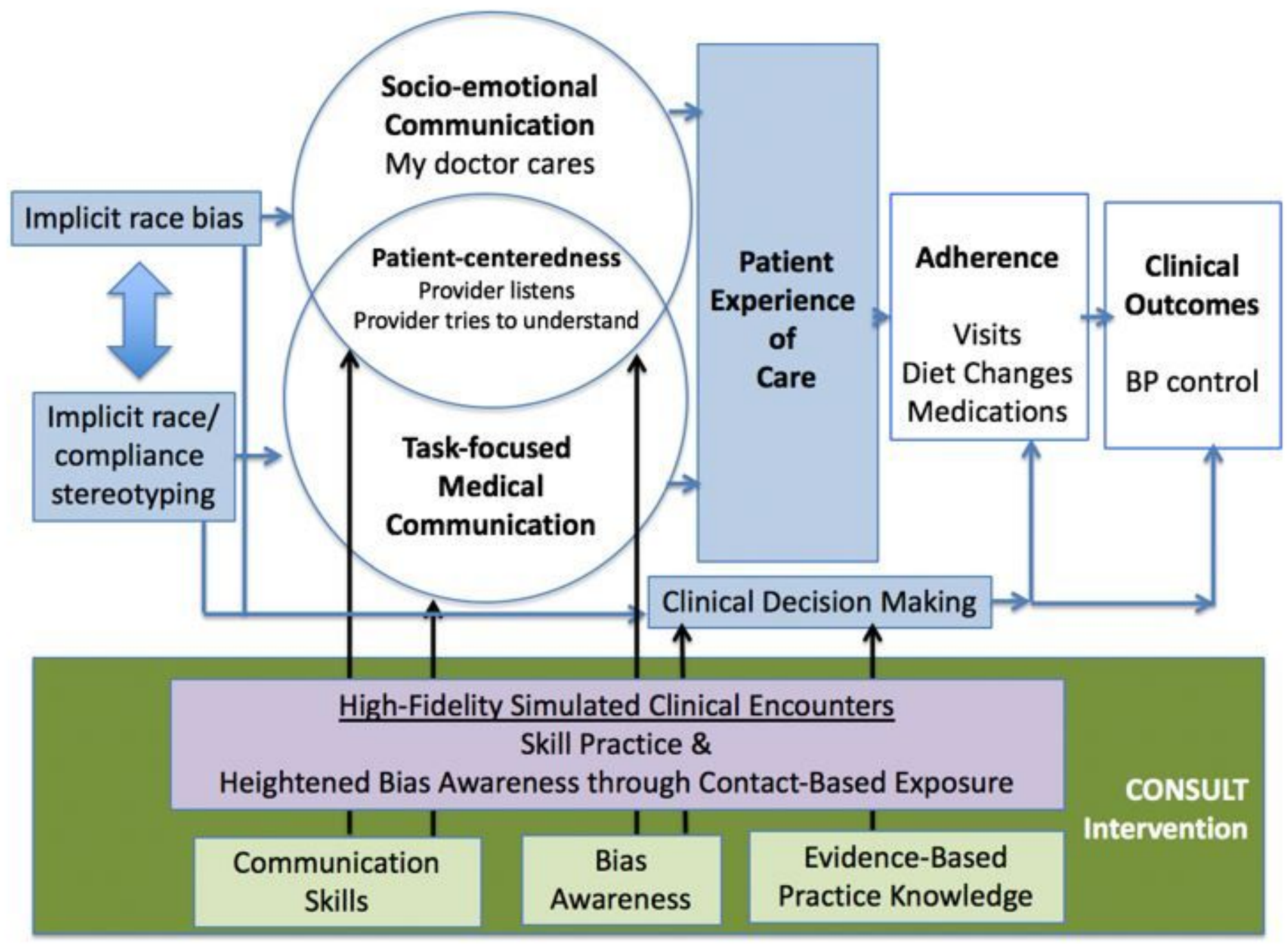

Figure 1

The CONSULT-BP intervention model 


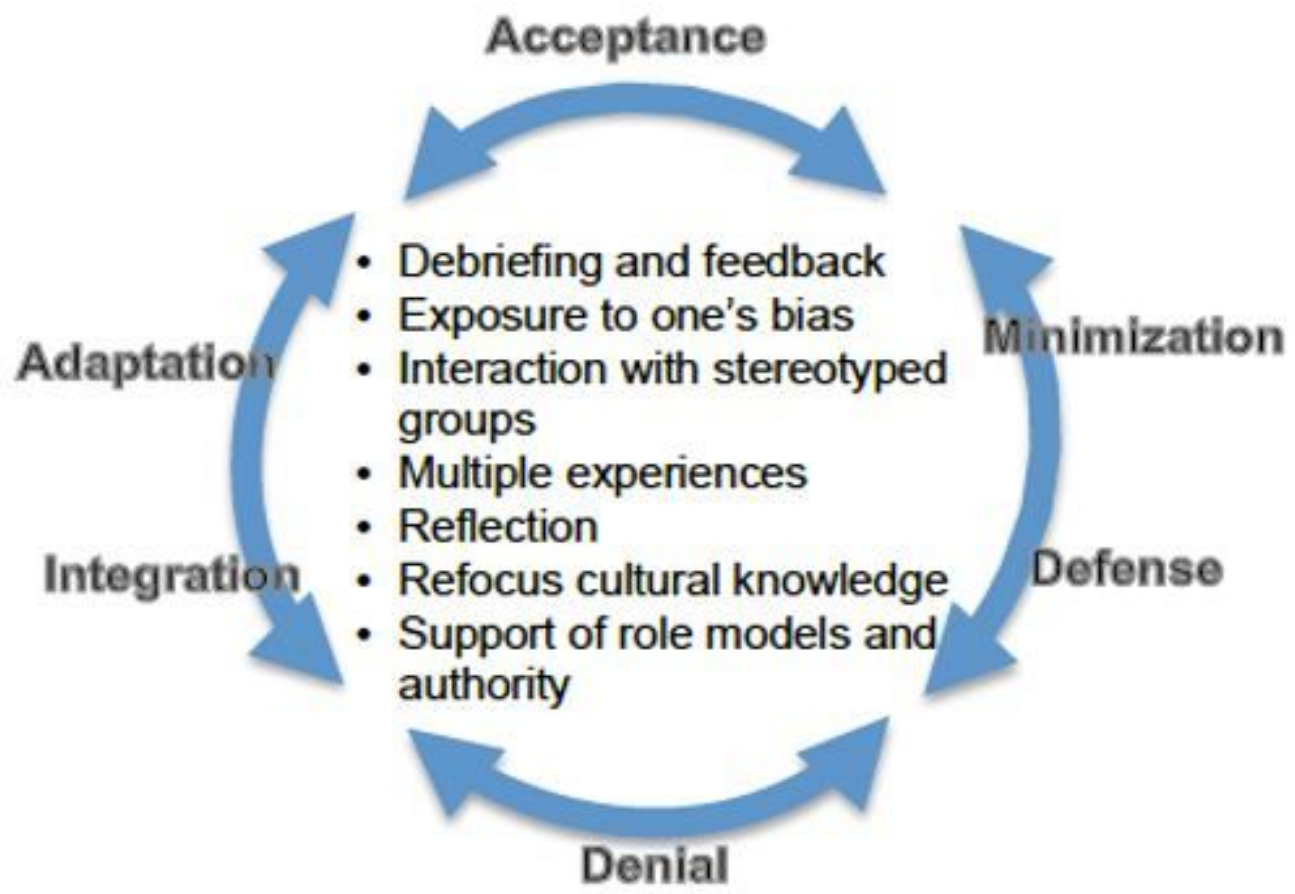

Figure 2

Adaptation of Bennett's intercultural competency theoretical framework24,25

\section{Supplementary Files}

This is a list of supplementary files associated with this preprint. Click to download.

- TjiaCONSULTProtocolBMCMedEdrevision10202020.docx 